\title{
EL AISLAMIENTO ACUSTICO EN EL SISTEMA DRAGADOS-PLASTBAU
}

Antonio Moreno, Instituto de Acústica/CSIC

José Antonio Comas, Dragados y Construcciones, S. A.

\section{OBJETIVOS}

Los estudios y ensayos acústicos, previstos en el programa de investigación del sistema Dragados-Plastbau, fueron realizados por la U.E.I. Radiación y Propagación Acústicas del Instituto de Acústica (C.S.I.C.) con los siguientes objetivos:

1.0 Comprobar el cumplimiento de la Norma NBE-CA-82 de cada uno de los elementos que forman el sistema Dragados-Plastbau.

2.0 En los casos en que no se alcanzaran los mínimos exigidos por la Norma, ensayar los complementos necesarios para su cumplimiento, atendiendo a razones de economía y de técnica constructiva.

Dadas las caracteristicas de las fórmulas de cálculo del aislamiento acústico de elementos no homogéneos, principalmente el grado de precisión, se planteó la investigación desde el punto de vista experimental.

Esta vía presenta además la ventaja de incluir las condiciones de montaje reales, imposibles de introducir en los modelos teóricos.

\section{DESCRIPCION DEL PROCEDIMIENTO DE ENSAYO}

Los ensayos fueron realizados en las instalaciones que el Instituto tiene en la calle Serrano de Madrid.

Los ensayos de aislamiento a ruido aéreo de los muros se llevaron a cabo, como se indica en la figura 1, en las cámaras de transmisión sin efecto de flancos que el Instituto tiene para este fin. Están formadas por compartimentos paralelepipédicos rectangulares de $100 \mathrm{~m}^{3}$ aproximadamente cada uno, adosadas a través de un anillo autoportante, soporte de la muestra, sin conexión rigida con las cámaras.

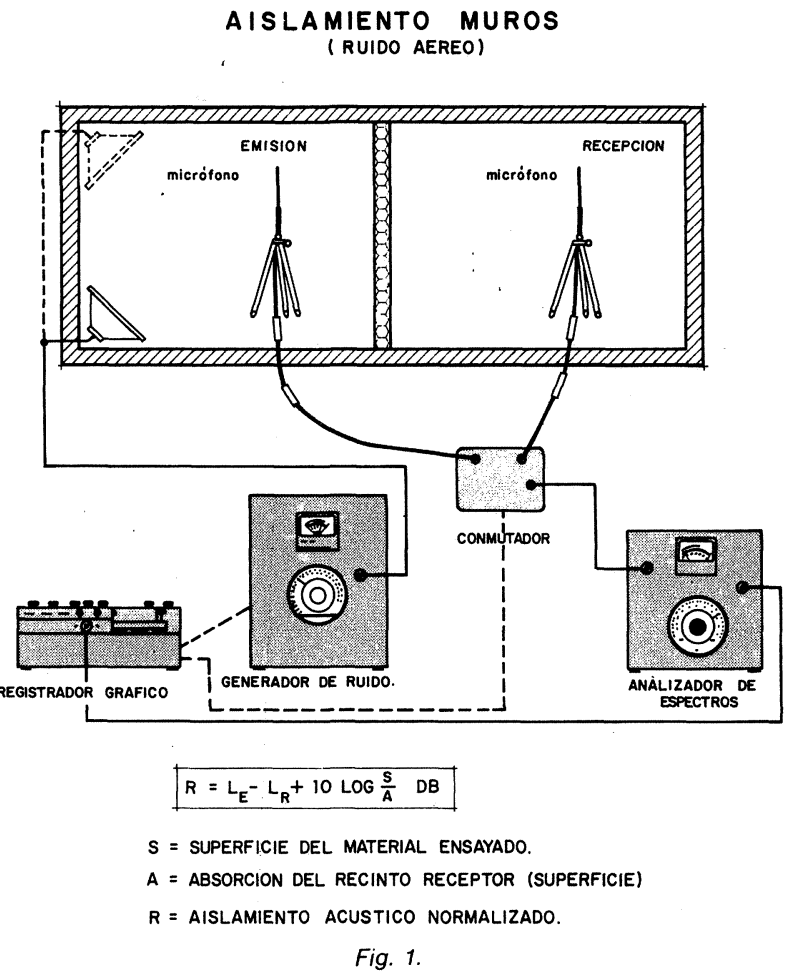

Los valores de $L_{E}$ y $L_{R}$ en estos ensayos se refieren a los niveles medios de presión acústica medidos, en la emisión y recepción, para una excitación con ruido blanco filtrado en tercios de octava.

Los ensayos de aislamiento a ruido aéreo y a ruido de impacto de los forjados fueron llevados a cabo en las cámaras de transmisión que el Instituto tiene para este fin (figura 2), formadas por dos compartimentos paralelepipédicos rectangulares de $50 \mathrm{~m}^{3}$ aproximadamente cada uno, adosadas a través del forjado en estudio y con las paredes sin independizar.

El valor de $L$ utilizado en estos ensayos se refiere al nivel de presión sonora en la cámara de re- 
AISLAMIENTO FORJADOS (RUIDOS DE IMPACTOS)

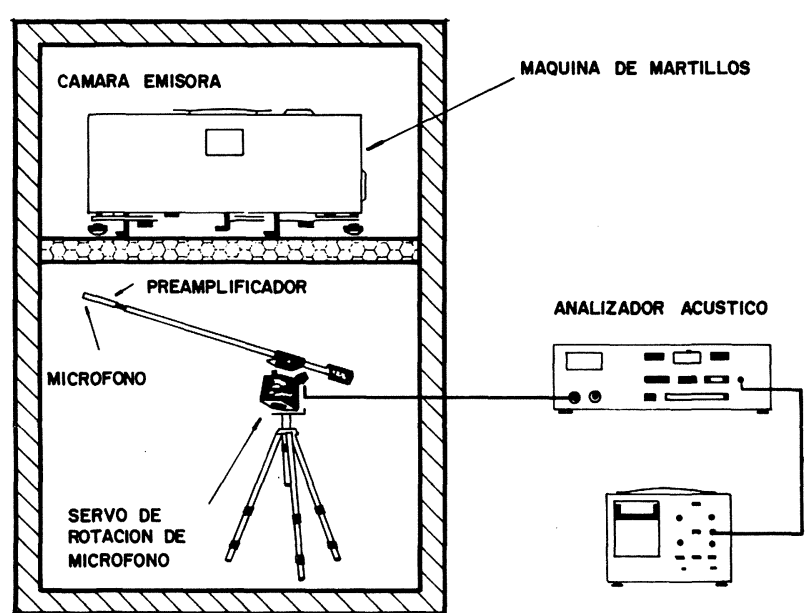

REGISTRADOR DE NIVEL

$$
L_{n}=L-10 \operatorname{LOg} \frac{10}{A} D B
$$

$A=$ ABSORCION DEL RECINTO EN M2.

$L_{n}=$ NIVEL DE RUIDOS DE IMPACTO NORMALIZADO

Fig. 2.

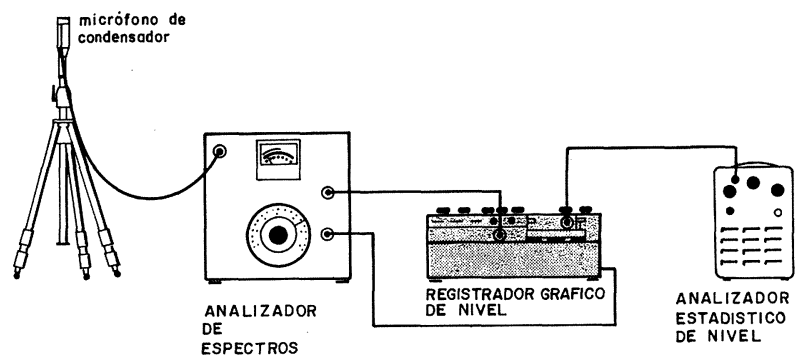

Fig. 3. - Detalle de la cadena de recepción, con analizador estadistico de nivel para la prueba de tiempo de reverberación.

cepción, filtrado en tercios de octava, producido por una máquina de martillos que cumple las especificaciones de la norma UNE-74040.

Para la medida de la absorción $A$ (figura 3 ) se ha seguido un procedimiento de medida que satisface las exigencias de la norma UNE-74041 (equivalente a la ISO-354).

El método de medida utilizado en todos los ensayos, tanto para muros como para forjados, satisface la norma UNE-74040 (equivalente a la norma ISO-140), tanto en lo relativo a las caracteristicas de las cámaras de transmisión como a la instrumentación para la generación, captación y análisis de los campos acústicos, o a la valoración y presentación de los resultados.

\section{ENSAYO DE LOS MUROS DRAGADOS-PLASTBAU}

Para cada uno de los muros ensayados se determinó la variación frecuencial o curva del aisla- miento a ruido aéreo, en tercios de octava. A partir de estos valores experimentales se determinaron los siguientes indices globales:

- aislamiento medio en $\mathrm{dB}$,

- aislamiento ponderado en dBA,

- indice $R_{W}$ (según ISO-717).

El índice de aislamiento en $\mathrm{dBA}$ es el que contempla la norma NBE-CA-82 del MOPU, vigente en nuestro país y equivale al indice francés; el indice $R_{w}$ es el usado en varios paises de la CEE y está ampliamente difundido.

El grado de detalle de los resultados obtenidos ha permitido valorar la influencia de los diversos factores que intervienen, tales como el espesor de las capas de microhormigón o la adición de un trasdosado en una o en ambas caras.

El poliestireno expandido empleado en todos los casos fue de la clase III.

En los ensayos correspondientes a los muros Dragados-Platsbau II los huecos correspondientes a los pilares no se llenaron con hormigón.

Cada muestra era de 4,50 m de ancho $\times 3,00 \mathrm{~m}$ de alto. Se construyeron con paneles de suelo a techo y las juntas verticales entre paneles fueron realizadas conforme a la tecnologia del sistema.

\subsection{Aislamiento a ruido aéreo muros Dragados-Plastbau I}

\section{Incidencia del espesor del recubrimiento}

(figura 4)

- Se comprobó que la frecuencia de resonancia (500 Hz), de mínimo aislamiento, no se modifica sustancialmente el aumentar el espesor de las capas, siendo el incremento de aislamiento (4dBA para $1 \mathrm{~cm}$ ) algo mayor de lo que se podría suponer conforme a la ley de masas.

- Para el espesor de 3,5 cms de microhormigón no se produce la disminución de aislamiento a $125 \mathrm{~Hz}$, que aparece con 2,5 cms, mientras que la caida del aislamiento entre $250 \mathrm{~Hz}$ y $500 \mathrm{~Hz}$ es similar.

\section{Incidencia del trasdosado (figura 5)}

- También en estos ensayos se presenta la frecuencia de resonancia a $500 \mathrm{~Hz}$ en todos los casos.

- Se comprobó la poca eficacia de trasdosar por una cara con Pladur $10 \mathrm{~mm}$ (ensayos 2 y 5), ya que el aumento del coste no justifica $1 \mathrm{dBA}$ de aumento de aislamiento.

- El trasdosar por una cara con Pladurbel 10 + 25 da buen resultado (ensayos 2 y 4), contrariamente a trasdosar con el mismo material por ambas caras (ensayos 3 y 4 ), ya que el aumento que se obtiene es de sólo $2 \mathrm{dBA}$. 


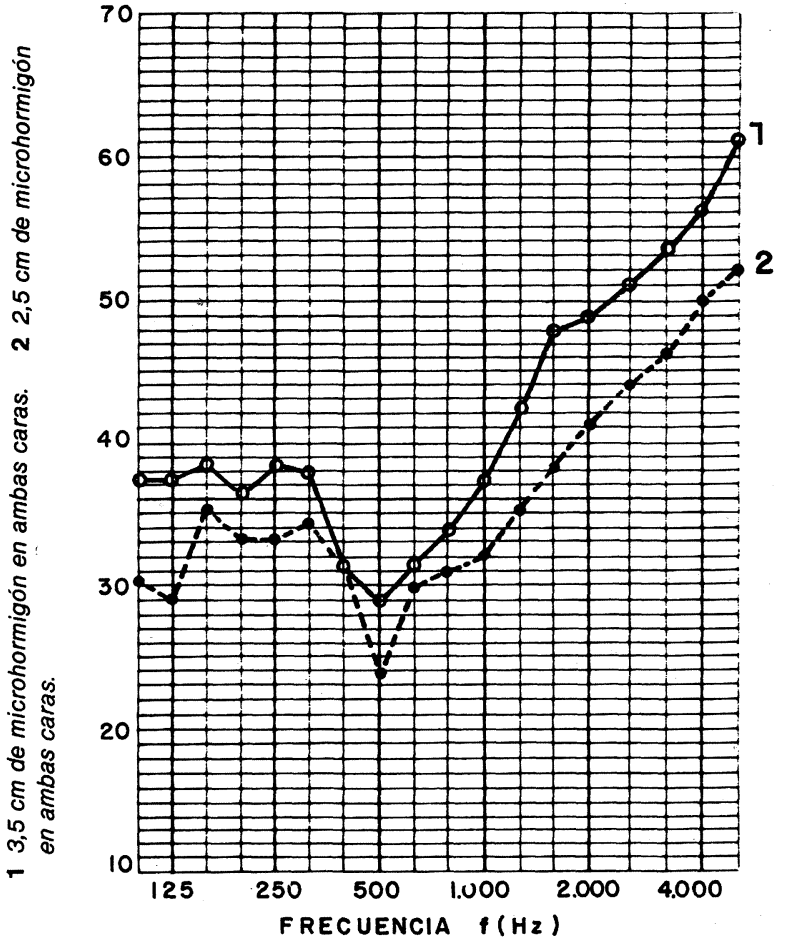

Fig. 4.

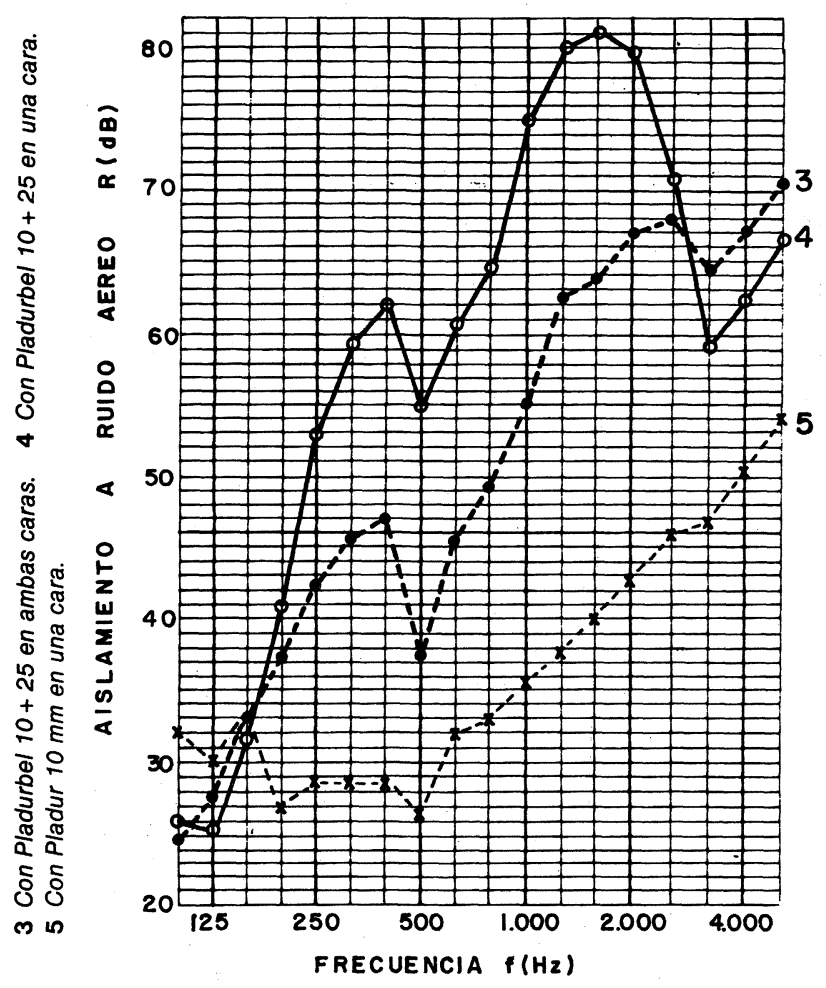

Fig. 5.

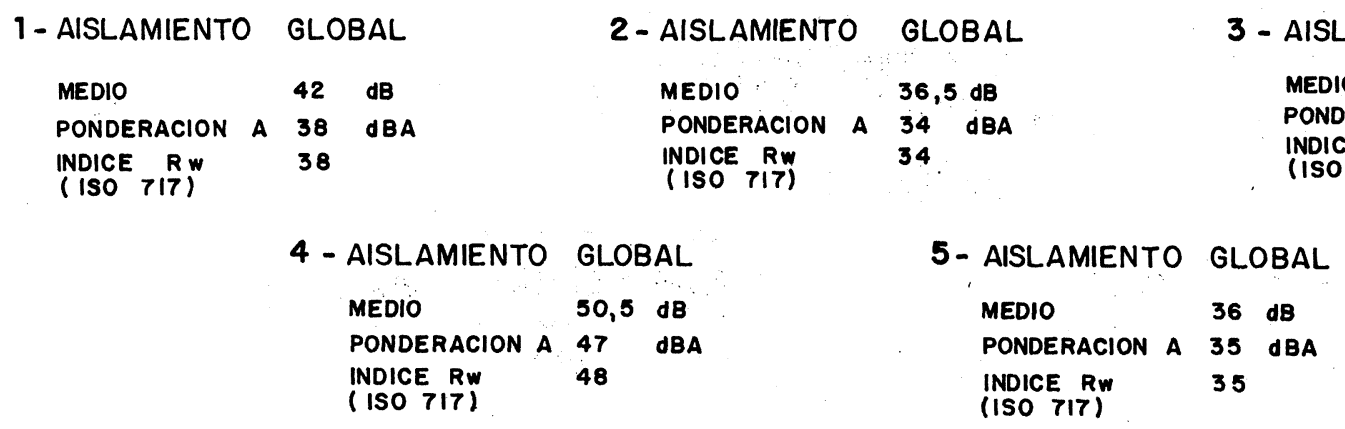

\subsection{Aislamiento a ruido aéreo muros Dragados-Plastbau II}

Incidencia del espesor de recubrimiento (figura 6)

- Al comparar los ensayos 6 y 8 se comprobó la nula eficacia, desde el punto de vista de aislamiento ponderado, de guarnecer los muros del tipo II con yeso, ya que siendo mejor su comportamiento para las frecuencias superiores a los $1.000 \mathrm{~Hz}$, por debajo de esta frecuencia es igual o inferior.

- De los ensayos 6 y 7 se confirma que es preferible enfoscar $2 \mathrm{~cm}$ por una de las caras que guarnecer $1,5 \mathrm{~cm}$ por cada cara del muro.

- De los resultados de los ensayos 7 y 8 queda demostrado que se obtiene un incremento de aislamiento al regruesar una de las caras de microhormigón en una proporción de 2,5 dBA para $2 \mathrm{~cm}$ de aumento, inferior a la que se obtuvo con los muros del tipo I (4 dBA para $1 \mathrm{~cm}$ por cada cara). Ello hace suponer que se ha- bría obtenido igual o superior aislamiento incrementando $1 \mathrm{~cm}$ el espesor de cada cara.

- La frecuencia de resonancia es de $125 \mathrm{~Hz}$ aproximadamente en todos los casos, inferior por lo tanto a la de los muros tipo I, al ser menor la rigidez de la conexión y mayor la separación entre las hojas de hormigón.

\section{Incidencia del trasdosado (figura 7)}

- De los resultados de los ensayos 10 y 11 se concluye que el disponer Pladur $10 \mathrm{~mm}$ en ambas caras no compensa el incremento de aislamiento. Tampoco compensa el colocar una sola cara con Pladur $10 \mathrm{~mm}$, a la vista de los ensayos 8 y 10.

- La solución de trasdosar una cara con Pladurbel $10+25$ se demuestra eficaz al comparar los ensayos 8 y 9.

- La frecuencia de resonancia se conserva en todos los casos próxima a los $125 \mathrm{~Hz}$. 

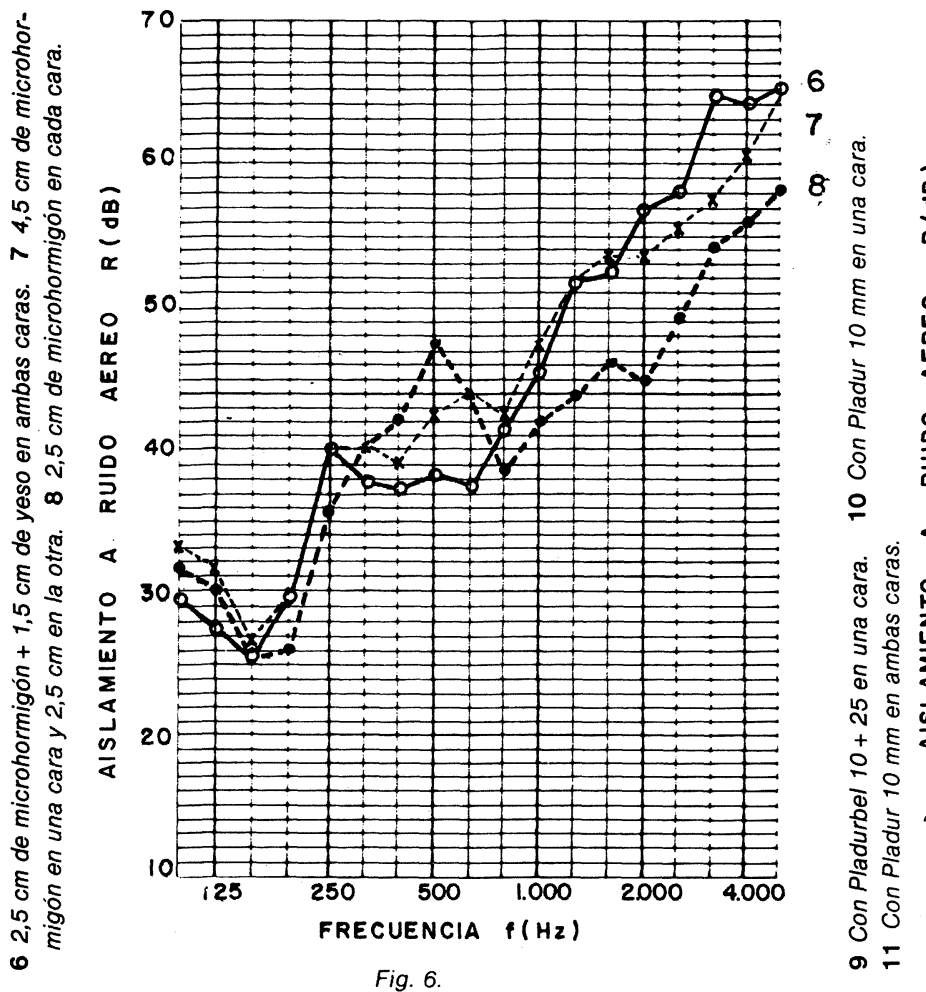

6 - AISLAMIENTO GLOBAL

$\begin{array}{ll}\text { MEDIO } & 44,5 \mathrm{~dB} \\ \text { PONDERACION A } & 42,5 \mathrm{dBA} \\ \text { INDICE RW } & 43 \\ \text { (ISO 7I7) } & \\ & \\ \text { - AISLAMIENTO } & \text { GLOBAL } \\ \text { MEDIO } & 59,5 \mathrm{~dB} \\ \text { PONDERACION A } & 49,5 \mathrm{dBA} \\ \text { INDICE RW } & 53 \\ \text { (ISO 7IT) } & \end{array}$
7-AISLAMIENTO GLOBAL
MEDIO $45 \mathrm{~dB}$
PONDERACION A 44,5 dBA
INDICE RW
45

10 - AISLAMIENTO GLOBAL

$\begin{array}{lll}\text { MEDIO } & 44,5 \mathrm{~dB} \\ \text { PONDERACION A } & 43 & \mathrm{dBA} \\ \text { INDICE RW } & 44 \\ \text { (ISO TI7) } & & \end{array}$

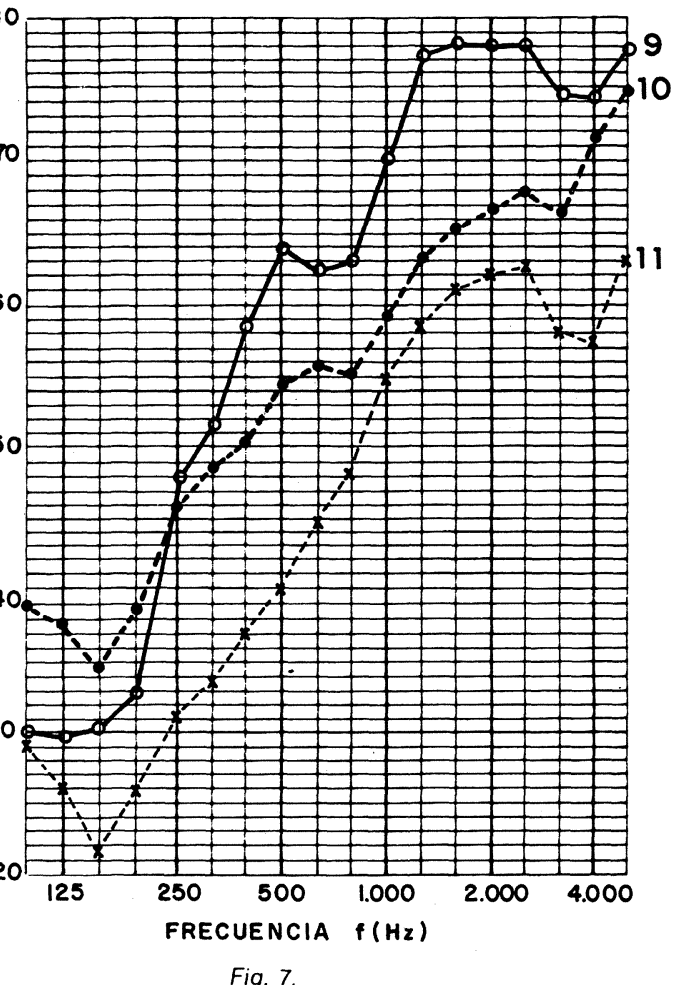

Fig. 7.

\subsection{Incidencia de la rigidez del núcleo en los muros, Dragados-Plastbau}

Con objeto de analizar el grado de influencia de la rigidez ofrecida por el poliestireno en la frecuencia de resonancia fundamental en los muros del tipo I y II, dado que el núcleo del tipo II está formado por 2 piezas independientes, se procedió a realizar el siguiente ensayo (figura 8 ).

Al comparar los resultados de los ensayos 2 (correspondiente al tipo I), 8 (correspondiente al tipo II) y 12 (hibrido del muro I y II) se pudo comprobar que los resultados del 12 son intermedios entre los obtenidos en el 2 y 8 :

- El aislamiento global es de $39 \mathrm{dBA}$, intermedio entre $34 \mathrm{dBA}$ (muro tipo I) y $42 \mathrm{dBA}$ (muro tipo II).
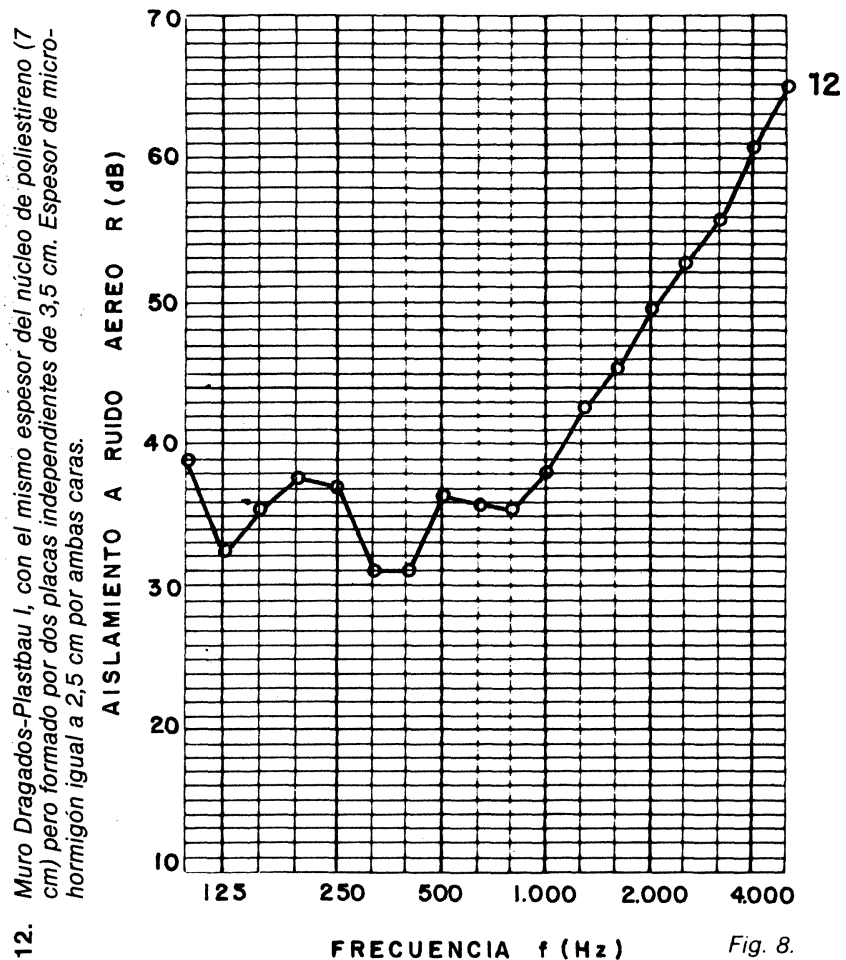
- La frecuencia de resonancia, que inicialmente era $500 \mathrm{~Hz}$ (la correspondiente al tipo I), muestra un desplazamiento hacia las bajas frecuencias, alcanzando un valor intermedio entre esta frecuencia y la de $250 \mathrm{~Hz}$.
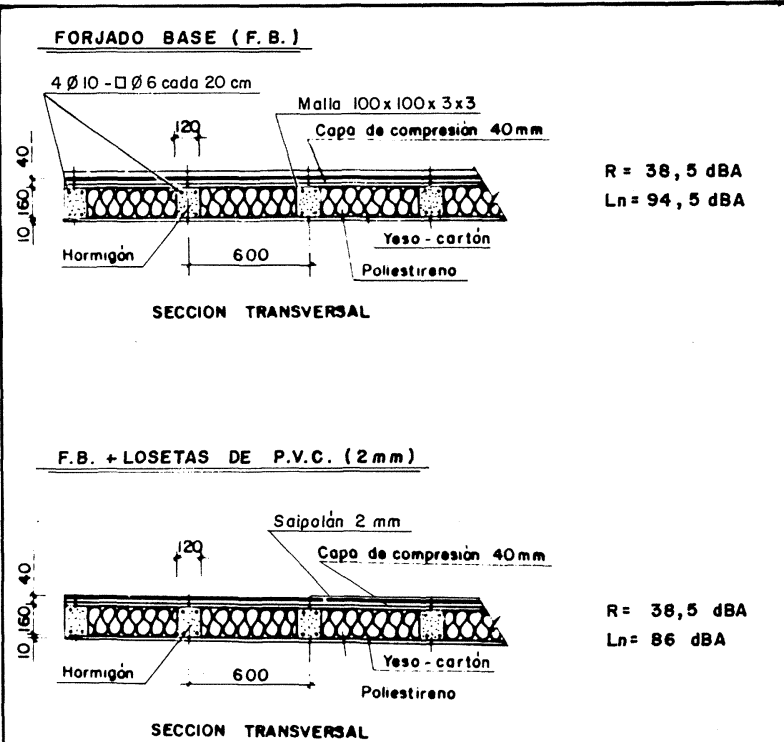

F.B + MOQUETA PUNZONADA $(3 \mathrm{~mm})$

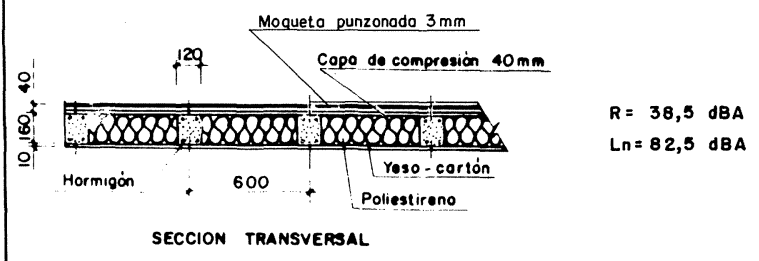

F.B + MOQUETA DE BUCLE CON ESPUMA DE POLIURETANO $(7 \mathrm{~mm})$

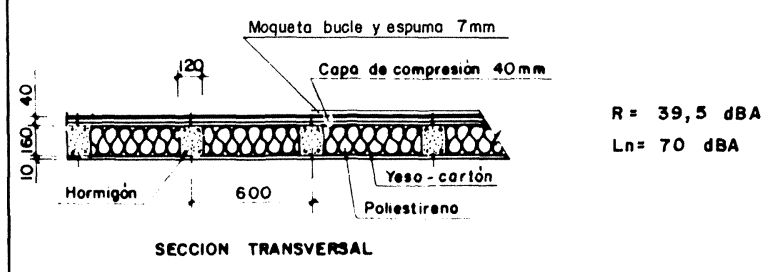

F.B. + TERRAZO FLotante s/LAMina "FOMPEX" $\left(5 \mathrm{~mm}\right.$ y $\left.50 \mathrm{~kg} / \mathrm{m}^{3}\right)$

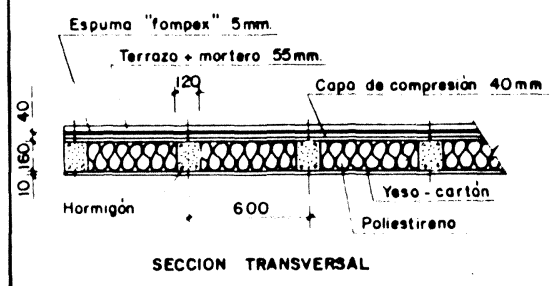

$R=47,5 \mathrm{dBA}$

$\operatorname{Ln}=81,5 \mathrm{dBA}(\sin$ capo nivolodora $L n=75$ dBA (con capo nivelodoro

Fig. 9.
Todo ello parece demostrar la incidencia que la rigidez del núcleo de poliestireno tiene en la capacidad aislante del muro.

Por otra parte la distinta longitud de los conectores en los sistemas I y II y la consiguiente diferente rigidización entre las capas de microhormigón, asi como el distinto ancho del núcleo de poliestireno, parece tener también alguna importancia a la vista de los $3 \mathrm{dBA}$ que no se llegan a alcanzar respecto del tipo II.

\section{ENSAYOS DE FORJADO}

El sistema Dragados-Plastbau puede emplear paneles del tipo I como cubierta de edificios. Sin embargo estas cubiertas no son visitables por lo que no están sometidas, de acuerdo con la norma NBE-CA-82, a cumplimiento de aislamiento a ruido de impacto.

Por ello los ensayos de forjados se han centrado sobre el forjado tipo "Cádiz" ideado por Dragados y Construcciones para el sistema DragadosPlastbau, terminado con diferentes solados.

En las soluciones ensayadas se determinó el espectro de frecuencias del aislamiento a ruido aéreo y de impacto, en tercios de octava y en octavas, asi como los valores globales correspondientes a:

- aislamiento medio en $\mathrm{dB}$

- aislamiento $R$ ponderado en $\mathrm{dBA}$

- indice $R_{W}$ (según ISO 717)

- indice $L_{n}$, w de ruidos de impacto (según ISO 717)

- nivel $L_{n}$ de ruidos de impacto en $d B A$

Cada muestra de forjado tenia unas dimensiones de $3,50 \mathrm{~m}$ de ancho $\times 4,60 \mathrm{~m}$ de largo, con las viguetas dispuestas en el sentido del ancho.

El poliestireno expandido empleado como bovedillas fue de la clase II.

Debido a la existencia de transmisiones indirectas en los ensayos a ruido aéreo, a efectos de aplicación de la Norma NBE-CA-82 los valores del aislamiento global en dBA correspondientes a estos ensayos se han incrementado en 2 puntos.

Lòs resultados que se obtuvieron quedan reflejados en la fig. 9. 
5. COMPARACION CON LOS SISTEMAS TRADICIONALES Y CUMPLIMIENTO DE LA NBE-CA-82

\subsection{Cerramientos exteriores}

Norma NBE-CA-82: Aislamiento global (mínimo) $=30$ dBA (INCLUIDOS HUECOS DE VENTANA)

\begin{tabular}{|c|c|c|}
\hline TIPO & CERRAMIENTO TRADICIONAL & $\begin{array}{c}\text { RUIDO AEREO } \\
\text { dBA }\end{array}$ \\
\hline 1 & $\begin{array}{l}15 \mathrm{cms} \text {. hormigón armado. } \\
2 \mathrm{cms} \text {. poliestireno } 11 \\
4 \mathrm{cms} \text {. ladrillo } \mathrm{H} / 5 \\
1,5 \mathrm{cms} \text {.enlucido yeso }\end{array}$ & $R=55$ \\
\hline 2 & $\begin{array}{l}18 \mathrm{cms} \text {. hormigón armado } \\
3 \mathrm{cms} \text {.poliestireno } 11 \\
4 \mathrm{cms} \text {. ladrillo H/s } \\
1,5 \mathrm{cms} \text {. enlucido yeso. }\end{array}$ & $R=57$ \\
\hline 3 & $\begin{array}{l}12 \text { cms.hormigón armado } \\
1,5 \text { cms.vidrio celular }\end{array}$ & $R=48$ \\
\hline 4 & $\begin{array}{l}2 \mathrm{cms} \text {. enfoscado } \\
11 \mathrm{cms} \text {. ladrillo H/D } \\
2 \mathrm{cms} \text {. enfoscado } \\
3 \mathrm{cms} \text {. poliestireno II } \\
4 \mathrm{cms} \text { ladrillo H/S } \\
1,5 \mathrm{cms} \text {. enlucido yeso. }\end{array}$ & $R=40$ \\
\hline 5 & $\begin{array}{l}11 \mathrm{cms} . l a d r i l l o ~ m a c i z o \\
4 \mathrm{cms} \text { poliestireno } 11 \\
4 \mathrm{cms} \text {.ladrillo } \mathrm{H} / \mathrm{S} \\
1,5 \mathrm{cms} \text {.enlucido yeso }\end{array}$ & $R=46$ \\
\hline $1 / 1$ & MUROS DP-1 & \\
\hline 1 & $\begin{array}{l}\text { Con } 2,5 \text { cms.de microhormigón en cada } \\
\text { cara. }\end{array}$ & $R=34$ \\
\hline 2 & $\begin{array}{l}\text { Con 3,5 cms, de microhormigón en cada } \\
\text { cara. }\end{array}$ & $R=38$ \\
\hline 3 & $\begin{array}{l}\text { El } 1 \text { trasdosado con Pladurbel } 10+25- \\
\text { en una cara. }\end{array}$ & $R=47$ \\
\hline 171 & MUROS DP-11 & \\
\hline 1 & $\begin{array}{l}\text { Con } 2,5 \text { cms.de microhormigón en cada } \\
\text { cara. }\end{array}$ & $R=42$ \\
\hline 2 & $\begin{array}{l}\text { El l regruesado con } 2 \mathrm{cms} \text {. de microhor- } \\
\text { migón en una cara. }\end{array}$ & $R=44,5$ \\
\hline 3 & $\begin{array}{l}\text { El } 1 \text { trasdosado con Pladurbel } 10+25 \text { en } \\
\text { una cara. }\end{array}$ & $R=49,5$ \\
\hline
\end{tabular}

\subsection{Tabiquería}

Norma NBE-CA-82: Tabiques interiores (minimo) 30/35 dBA. Tabiques de separación (mínimo) 45 dBA

\begin{tabular}{|c|c|c|}
\hline TIPO & TABIQUERIA TRADICIONAL & $\begin{array}{l}\text { RUIDO AEREO } \\
(\mathrm{dBA})\end{array}$ \\
\hline 1 & $\begin{array}{l}4 \text { cms. ladrillo H/S } \\
1,5 \text { cms. enlucido yeso (cada cara) }\end{array}$ & $R=32$ \\
\hline 2 & $\begin{array}{l}9 \text { cms.ladrillo } \mathrm{H} / \mathrm{S} \\
1,5 \mathrm{cms} \text {.enlucido yeso (cada cara) }\end{array}$ & $R=35$ \\
\hline 3 & $\begin{array}{l}11 \mathrm{cms} \text { ladrillo macizo } \\
1,5 \mathrm{cms} \text { enlucido yeso (cada cara) }\end{array}$ & $R=46$ \\
\hline 4 & 13 cmṣ.hormigón armado & $R=50$ \\
\hline 5 & $\begin{array}{l}6 \text { cmsid escayola } \\
8 \text { cmsi: escayola } \\
10 \mathrm{cms} \text { escayola }\end{array}$ & $\begin{array}{l}R=32 \\
R=36 \\
R=39\end{array}$ \\
\hline 6 & 6 cms.pladur trillaje & $R=33$ \\
\hline 7 & $\begin{array}{l}72 \text { cms. pladurmetal } \\
(13+46+13)\end{array}$ & $\begin{array}{l}R=40(\operatorname{Sinf} \cdot V) \\
R=43(\operatorname{Conf} \cdot V)\end{array}$ \\
\hline 8 & $\begin{array}{l}100 \text { cms.pladurmetal } \\
(15+70+15)\end{array}$ & $\begin{array}{l}R=43(\text { Sinf. } \cdot V) \\
R=45(\text { Conf } \cdot V)\end{array}$ \\
\hline & MUROS DP-1 & \\
\hline 1 & $\begin{array}{l}\text { Con } 2,5 \text { cmside microhormigón } \\
\text { en cada cara. }\end{array}$ & $R=34$ \\
\hline 2 & $\begin{array}{l}\text { Con } 3,5 \text { cms. de microhormigón } \\
\text { en cada cara. }\end{array}$ & $R=38$ \\
\hline 3 & $\begin{array}{l}\text { El l trasdosado con Pladurbel } \\
10+25 \text { en una cara. }\end{array}$ & $R=47$ \\
\hline & MUROS DP-11 & \\
\hline 1 & $\begin{array}{l}\text { Con } 2,5 \text { cms. de microhormigón en } \\
\text { cada cara. }\end{array}$ & $R=42$ \\
\hline 2 & $\begin{array}{l}\text { El } 1 \text { regruesado con } 2 \text { cms.. de mi- } \\
\text { crohormigón en una cara. }\end{array}$ & $R=44,5$ \\
\hline 3 & $\begin{array}{l}\text { El } 1 \text { trasdosado con Pladurbel -- } \\
10+25 \text { en una cara. }\end{array}$ & $R=49,5$ \\
\hline
\end{tabular}




\subsection{Forjados}

Norma NBE-CA-82: Aislamiento a ruido aéreo (minimo) 45 dBA. Aislamiento a ruido de impacto (mínimo) $80 \mathrm{dBA}$

\begin{tabular}{|c|c|c|c|}
\hline \multirow[t]{2}{*}{ TIPO } & \multirow[t]{2}{*}{ FORJADO TRADICIONAL } & \multirow{2}{*}{\multicolumn{2}{|c|}{\begin{tabular}{|c|c} 
AISLAMIENTO & ACUST ICO(NBE-CA-82) \\
AEREO DBA & IMPACTO DBA \\
\end{tabular}}} \\
\hline & & & \\
\hline 1 & $\begin{array}{l}\text { Unidireccional de } 20 \mathrm{~cm} \text { con bo } \\
\text { vedilla cerámica y solado de } 80 \\
\mathrm{~kg} / \mathrm{m} 2 \text { con } 1,5 \mathrm{cms} \text {.de enlucido - } \\
\text { de yeso. }\end{array}$ & $R=48$ & $L_{N}=87$ \\
\hline 2 & $\begin{array}{l}\text { Losa de hormigón armado de } 20 \text { - } \\
\text { cmśr con solado de } 80 \mathrm{~kg} \sqrt{ } / \mathrm{m} 2 \text {. }\end{array}$ & $R=59$ & $L_{N}=76$ \\
\hline 3 & $\begin{array}{l}\text { For jado reticular de } 20 \text { cms.con } \\
\text { bovedilla cerámica y solado de } \\
80 \mathrm{~kg} / \mathrm{m} 2 \text { con } 1,5 \text { cmşde enluci- } \\
\text { do de yeso. }\end{array}$ & $R=50$ & $\mathrm{~L}_{\mathrm{N}}=85$ \\
\hline & FORJADO DRAGADOS-PLASTBAU & & \\
\hline 1 & $\begin{array}{l}\text { Capa de compresión de } 4 \mathrm{cms} \text {, can } \\
\text { to nervio } 16 \mathrm{cms} \text {, con placa } \mathrm{N}-1 \overline{0} \\
\text { y } 60 \mathrm{cmss} \text { entre ejes de nervios } \\
\text { de } 12 \mathrm{~cm} \$ \text {. }\end{array}$ & $R=38,5$ & $L_{N}=94,5$ \\
\hline 2 & $\begin{array}{l}\text { E1 mismo con moqueta Saipolan } \\
(2 \mathrm{~mm}) \text {. }\end{array}$ & $R=38,5$ & $\mathrm{~L}_{\mathrm{N}}=86$ \\
\hline 3 & $\begin{array}{l}\text { El mismo con moqueta punzonada } \\
(3 \mathrm{~mm}) \text {. }\end{array}$ & $R=38,5$ & $L_{N}=82,5$ \\
\hline 4 & $\begin{array}{l}\text { El mismo con moqueta de bucle - } \\
\text { de espuma de poliuretano }(7 \mathrm{~mm})\end{array}$ & $R=39,5 *$ & $L_{N}=70$ \\
\hline 5 & $\begin{array}{l}\text { El mismo con pavimento de terra } \\
\text { zo flotante sobre lámina fonpex } \\
\text { de } 5 \mathrm{~mm} \text { y } 50 \mathrm{~kg} / \mathrm{m} 3 \text {. }\end{array}$ & $R=47,5$ & $L_{N}=75$ \\
\hline 6 & $\begin{array}{l}\text { El mismo con pavimento de terra } \\
\text { zo sin flotar. }\end{array}$ & $R=45 *$ & $\mathrm{~L}_{\mathrm{N}}=85^{*}$ \\
\hline
\end{tabular}

* DATOS EST IMADOS

\subsection{Conclusiones}

1.0 Una fachada es un elemento mixto, formado por huecos y parte ciega, donde los ele- mentos más débiles, acústicamente hablando, son las ventanas.

Por otra parte es conocido que el aislamiento global de un elemento constructivo mixto es, como máximo, $10 \mathrm{dBA}$ mayor que el del elemento constructivo más débil (NBE-CA82. APARTADO 1.36).

En conclusión, basta con disponer de un aislamiento acústico en parte ciega de 40 dBA para tener la seguridad de cumplir la norma en todos los casos en que el porcentaje de huecos sea $\leqslant 25 \%$.

Por lo tanto todas las soluciones de muros Dragados-Plastbau que tengan un aislamiento acústico igual o superior a $40 \mathrm{dBA}$, son válidas sin necesidad de análisis.

2.0 Todos los muros Dragados-Plastbau cumplen la norma para tabiques interiores.

3.0 Los muros Dragados-Plastbau I tienen que ser trasdosados para poder ser empleados como elementos de separación con zonas comunes o con otras viviendas.

4.0 Los muros Dragados-Plastbau II pueden ser utilizados como elementos de separación, siempre y cuando el espesor de las cáscaras de microhormigón sea igual a $3,5 \mathrm{~cm}$. En otro caso tendrán que ser trasdosados.

5.0 El forjado del sistema Dragados-Plastbau sólo cumple la norma de aislamiento aéreo y a impacto mediante un pavimento pesado y flotante, exactamente igual que los forjados tradicionales.

\section{publicación del i.e.t.c.c.}

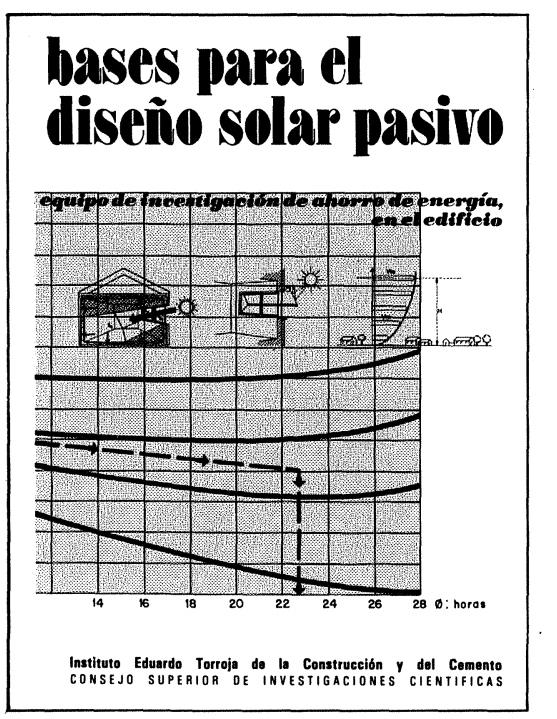

Equipo de Ahorro de Energía en el edificio

Dirección y coordinación: Arturo García Arroyo

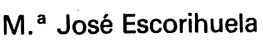

José Luis Esteban

José Miguel Frutos

Manuel Olaya

Bernardo Torroja
Las dificultades de suministro y el alto coste de los productos energéticos convencionales han despertado la atención de los usuarios, técnicos e industriales de la edificación hacia los procedimientos $y$ sistemas en que se basa cos procedimientos $y$ sistemas en que se basa Esto ha generado un rápido desarrollo industrial y comercial que, en opinión de los autores de este libro, arrastran los siguientes defectos: un mimético tecnologismo respecto de los sistemas convencionales que violenta las peculiaridades de la energia qolar (baja densidad y variabilidad en el tiempo), y una escasa
selectividad en la aplicación de los sistemas y procedimientos pasivos dando origen a un ecumenismo arquitectónico solar, al margen de las condiciones climáticas y funcionales especificas de cada caso y lugar.

En este libro, utilizando criterios y metodologia pedagógicos, se dan los fundamentos e instrumentos teórico-prácticos necesarios para el planteamiento de todo proyecto arquitectónico solar pasivo, de acuerdo con los principios éticos y económicos de conservación y ahorro de energía. Es decir: respeto de los presupuestos bioclimáticos, búsqueda de la máxima captación y acumulación de la radiación solar, y esmero en el aislamiento térmico de los cerramientos.

Un volumen encuadernado en cartulina ibiza plastificada, a cinco colores, de $16 \times 23 \mathrm{~cm}$, compuesto de 216 páginas, 217 figuras, 87 gráficos, 19 tablas y 10 cuadros. 